4.

Derecho procesal 

Revista de Derecho

de la Pontificia Universidad Católica de Valparaíso

XXXIV (Valparaíso, Chile, $1^{\text {er }}$ Semestre de 2010)

[pp. $505-520$ ]

\title{
L'ESECUZIONE PROCESSUALE INDIRETTA NELLA RIFORMA DEL “CODICE DI PROCEDURA CIVILE" ITALIANO DAL 2009
}

["The Indirect Execution of Judgement in the Reform of the Italian Code of Civil Procedure of 2009"]

\author{
Achille Saletti* \\ Universidad de Milán
}

\section{RESUMEN}

Este artículo desarrolla la reciente modificación al Codice di Procedura Civile italiano que introduce cambios radicales en la ejecución procesal civil con la introducción de las "astreintes" o medida coercitiva pecuniaria en beneficio de la contraparte. La disposición se ubica en la regulación de las obligaciones de hacer infungibles o de no hacer, pero no limita tampoco su aplicación para otro tipo de obligación.

Palabras Clave: Ejecución indirecta - Medidas coercitivas - "Astreintes".
ABSTRACT

This article explains the recent modification the Italian Codice di Procedura Civil underwent that includes radical changes in the civil execution of judgement and also includes the "penalty payments" or pecuniary coercive measure for the benefit of the counterparty. This stipulation is part of the regulation of obligations to undertake infungibles or not undertake them, but does not limit its application to other types of obligation.

KEYWORDS: Indirect execution Coercive measures - "Penalty payments".

* Profesor ordinario de Derecho procesal civil y Director del Instituto de Derecho Procesal Civil de la Universidad de Milán. Dirección postal: Via F.lli Gabba 7, 20121 Milano, Italia. Correo electrónico: achille.saletti@gmail.com. 


\section{L’ennesima novella del “Codice di Procedura Civile” L'ESECUZIONE PROCESSUALE INDIRETTA}

La legge 18 giugno 2009, n. 69, che ha realizzato l'ennesima novella del codice di procedura civile, tra le molteplici innovazioni introdotte, si caratterizza per aver previsto una nuovo potere in capo al giudice civile: infatti, nel titolo quarto del libro terzo del codice di rito, dedicato all'esecuzione forzata di obblighi di fare e di non fare, è stato inserito il nuovo articolo 614 bis, il quale, sotto la rubrica "Attuazione degli obblighi di fare infungibile o di non fare", sancisce che "con il provvedimento di condanna il giudice, salvo che ciò sia manifestamente iniquo, fissa, su richiesta di parte, la somma di denaro dovuta dall'obbligato per ogni violazione o inosservanza successiva, ovvero per ogni ritardo per l'esecuzione del provvedimento"1.

Il nostro legislatore ha introdotto, così, una forma di coazione indiretta, finalizzata ad ottenere l'esecuzione volontaria della sentenza del giudice: con ciò recependo, da un lato, l'auspicio della dottrina, che sottolineava da tempo l'esigenza dell'introduzione, in via generale, di misure coercitive indirette nel nostro sistema ${ }^{2}$; e realizzando, dall'altro, almeno in parte, le proposte contenute nei più recenti progetti di riforma organica del codice di procedura civile ${ }^{3}$. Cosi il nostro ordinamento si allinea a quelli stranieri, che un similare potere da tempo prevedono $\mathrm{s}^{4}$.

${ }^{1}$ Il primo comma dell'articolo prosegue sancendo che "il provvedimento di condanna costituisce titolo esecutivo per il pagamento delle somme dovute per ogni violazione o inosservanza. Le disposizioni di cui al presente comma non si applicano alle controversie di lavoro subordinato pubblico e privato e ai rapporti di collaborazione coordinata e continuativa di cui all'articolo 409". Il secondo comma stabilisce, ancora, che "il giudice determina l'ammontare della somma di cui al primo comma tenuto conto del valore della controversia, della natura della prestazione, del danno quantificato o prevedibile e di ogni altra circostanza utile".

${ }^{2}$ In argomento v. il fondamentale saggio di ChIARLONI, Misure coercitive e tutela dei diritti (Milano, 1980); e, per ogni opportuno riferimento, Silvestri - TARUfFo, Esecuzione forzata, III: Esecuzione forzata e misure coercitive, in Enc. giuridica, XIII, nonché la Postilla di aggiornamento a tale voce (2006), curata sempre da SILVESTRI.

${ }^{3}$ Sia il progetto di legge delega per la riforma del codice di procedura civile predisposto dalla Commissione ministeriale presieduta dal prof. Giusepppe Tarzia [che si può leggere in Riv. Dir. Proc. (1996), pp. 948 ss.], sia quello predisposto dalla Commissione ministeriale presieduta dal prof. Romano Vaccarella (trasfusa nel d.d.l. 4578, presentato nel corso della XVI Legislatura) prevedevano l'introduzione di forme generalizzate di coazione all'adempimento. In questo senso v., anche, da ultimo, Proto Pisani, Per un nuovo codice di procedura civile, in Foro it., 5 (2009), pp. 1 ss., spec. 99.

${ }^{4}$ Ciò accade, per esempio, in Francia e in Belgio con il sistema delle astreintes. In argomento v. da ultimo, Vullo, L'esecuzione indiretta tra Italia, Francia e Unione 


\section{II. ¿VALENZA GENERALE DELL'ARTICOLO, PER OGNI STATUIZIONE DI CONDANNA?}

Nonostante la sedes dell'articolo, non siamo di fronte ad un'attività esecutiva, bensì ad un potere che inerisce al processo di cognizione e all'attività di decisione sulle pretese di cui si chiede il riconoscimento, cui detto procedimento è deputato. La collocazione attribuita alla norma può essere, tuttavia, giustificata dal fatto che l'intento legislativo è stato quello di ottenere l'attuazione della sentenza in via volontaria'; e tradizionalmente si parla con riguardo alle misure coercitive indirette, di esecuzione processuale indiretta ${ }^{6}$.

La nuova previsione normativa lascia, però, dubbiosi circa la sua esatta portata. L'articolo, letto senza tener conto della rubrica, sembra avere una valenza generale, per ogni statuizione di condanna, a prescindere dall'obbligazione cui si riferisca. Se, però, si guarda alla rubrica, la conclusione è assolutamente differente, essere, cioè, disposizione destinata a trovar applicazione esclusivamente qualora oggetto di tali statuizioni siano delle obbligazioni di fare infungibile o di non fare.

Questa seconda soluzione appare più persuasiva ${ }^{7}$, se si considera, da un lato, la voluntas legis, chiaramente in questo senso, come risulta dalla già citata relazione al d.d.l. n. 14418; dall'altro, che all'identica conclusione induce la collocazione dell'articolo. Esso, come già si è notato, non è stato inserito tra le norme dedicate al processo di cognizione, ad es. nel capo del

europea, in Riv. Dir. Proc. (2004), pp. 727 ss. Sulle esperienze straniere di attuazione dei diritti, v., in generale, TARUFF, L'attuazione esecutiva dei diritti: profili comparatistici, in Riv. Trim. Dir. Proc. Civ. (1988), pp. 142 ss.

${ }^{5} \mathrm{Di}$ "coercizione indiretta per l'adempimento degli obblighi di fare infungibile e per gli obblighi di non fare" parla la relazione al d.d.l. n. 1441, poi trasfuso nella 1 . 18.6.2009, n. 69, sub articolo 54. Critici sulla collocazione in questione, Punzi, Novità legislative e ulteriori proposte di riforma in materia di processo civile, in Riv. Trim. Dir. Proc. Civ. (2008), p. 1203; RiccI, G. F., Ancora novità (non tutte importanti, non tutte pregevoli) sul processo civile, in Riv. Dir. Proc. (2008), p. 1362.

${ }^{6} \mathrm{Cfr}$. il classico lavoro di Ferrara, L., L'esecuzione processuale indiretta (Napoli, 1915); e, da ultimo, v. Mandrioli, Corso di diritto processuale civile (7a ediz., Torino, 2009), VII, p. 116.

${ }^{7}$ Conf. Mandrioli, ibid.; Ricci, G. F., La riforma del processo civile (Torino, 2009), p. 89.

${ }^{8}$ La quale, sub articolo 54, si esprime come segue: "La norma introduce uno strumento di coercizione indiretta per l'adempimento degli obblighi di fare infungibile e per gli obblighi di non fare, prevedendo che la sentenza che accoglie la domanda di condanna all'adempimento di tali obblighi contenga anche la determinazione di una somma di denaro spettante al creditore per ogni violazione o inosservanza successiva della pronuncia”. 
libro secondo dedicato alla decisione della causa, bensì, conformemente alla previsione della rubrica, nel titolo quarto del libro terzo, concernente in maniera specifica l'esecuzione degli obblighi di fare e di non fare. Collocazione che si può giustificare solo in quanto il potere del giudice di pronunciare l'ordine di pagamento sia circoscritto, appunto, ai casi di violazione delle obbligazioni in questione.

In conseguenza, se è sicuramente vero che, tramite la norma qui commentata, si è reso generale un potere di coercizione indiretta prima presente solo in via eccezionale ${ }^{9}$, è altrettanto vero che tale potere non inerisce alla tutela di condanna in quanto tale, ma concerne taluni specifici tipi di obbligazione soltanto.

Due le considerazioni che si possono trarre, in via preliminare, dal nuovo sistema.

In primo luogo, la tutela di condanna ha ormai acquisito un ambito di applicazione assai più ampio di quello che aveva sino ad ora. Se, infatti, secondo l'opinione dominante, tale tutela era, prima della riforma ${ }^{10}$, ammissibile solo allorquando fosse possibile l'esecuzione forzata della decisione del giudice, oggi questa correlazione è venuta meno: alla luce dell'articolo 614 bis c.p.c. è sicuramente possibile chiedere la condanna all'esecuzione di un'obbligazione infungibile, ancorché difetti la possibilità di sua realizzazione coattiva. Quindi la tutela di condanna, oggi, non incontra più, indiscutibilmente, i limiti del passato.

In secondo luogo, la norma in esame evidenzia che il legislatore si è sforzato, nel quadro delle forme di tutela apprestate dall'ordinamento, di realizzarne un'ulteriore per le obbligazioni considerate dalla norma in esame. Forma di tutela che si aggiunge a quella del risarcimento del danno, unica finora possibile per le obbligazioni infungibili; ma che anche concreta per talune obbligazioni di non fare, come vedremo, una forma di tutela aggiuntiva a quella derivante dalle possibilità di esecuzione in forma specifica.

\section{CATEGORIE DI OBBLIGAZIONI L'ESECUZIONE} PROCESSUALE INDIRETTA

La disposizione in esame appare dedicata a due categorie di obbliga-

\footnotetext{
${ }^{9}$ Per la disamina delle misure coercitive già presenti nel nostro sistema, ma relative a specifiche materie, v. SiLVESTRI, Postilla di aggiornamento, citata alla nota 1 .

${ }^{10}$ In proposito cfr., per tutti, MANDRIOLI, Sulla correlazione necessaria tra condanna ed eseguibilità forzata, in Riv. Trim. Dir. Proc. Civ. (1976), pp. 1342 ss. In giurisprudenza v. Cass., 6 febbraio 1999, n. 1037 (ma nel senso di superare la correlazione tra condanna ed esecuzione, cfr. Cass., 17 luglio 1992, n. 8721).
} 
zioni, di fare infungibile e di non fare: peraltro, con riferimento a questo secondo gruppo di obbligazioni, si possono avere delle perplessità se la nuova tutela si riferisca a tutte o solo ad alcune di esse ${ }^{11}$.

Nell'ambito delle obbligazioni di non fare se ne possono distinguere, infatti, di due tipi. Da un lato quelle che si esauriscono nell'obbligo di non tenere determinati comportamenti (ad es., il patto di non concorrenza), le quali, se violate, generano un obbligo risarcitorio, senza che vi sia la possibilità di ripristino in forma specifica. Accanto a queste obbligazioni, che si possono definire di non fare in senso stretto (o di non fare infungibile), se ne possono individuare delle altre, alla cui violazione segue la possibilità di ripristino in forma specifica: sicché, a differenza dalle prime, si può reintegrare completamente il diritto violato. È il caso, ad es., dell'edificazione di un edificio che non avrebbe potuto essere costruito, la cui distruzione consente di rimettere la situazione nel pristino stato (obbligazioni che si possono definire di "distruggere" o di "disfare").

Sussiste una notevole differenza tra le menzionate categorie, giacché le obbligazioni di non fare, che comportano un successivo obbligo di distruggere, sono obbligazioni fungibili, non essendovi ostacoli, se non in ipotesi eccezionali stabilite dalla legge (v. ad es., l'articolo $2933,2^{\circ}$ co., c.c.), all'eliminazione coattiva, nelle forme di cui agli articoli 612 e ss. c.p.c., di quanto non avrebbe dovuto essere fatto. Per contro, alla violazione delle obbligazioni di non fare in senso stretto non consegue la possibilità di ripristinare in forma specifica la situazione precedente, mediante la distruzione, sicché esse sono sanzionate meramente sul piano risarcitorio.

Vi è, dunque, una stretta affinità tra quest'ultima categoria e le obbligazioni di fare infungibile, che, a loro volta, non fruiscono di una tutela esecutiva, ma sino ad ora erano salvaguardate semplicemente dall'obbligo di risarcimento del danno; il che fa sorgere il dubbio se la previsione dell'articolo 614 bis c.p.c. valga per entrambe le categorie di non fare o solo per quella priva di salvaguardia esecutiva ed assimilabile, sul piano della tutela, all'obbligazione di fare infungibile.

La soluzione più ampia appare, però, preferibile. Giocano in questo senso sia la lettera della norma, ove si parla genericamente di obbligazioni di non fare (e la specificazione "infungibile" non si riferisce ad esse, ma solo a quelle di fare); sia la collocazione della stessa nel titolo dove sono disciplinate le obbligazioni di "distruggere". Se a ciò si aggiunge che la previsione del primo comma dell'articolo in esame, dove si parla

${ }^{11}$ Mandrioli, Corso, cit. (n. 6), p. 116; e Soldi, A., in Bucci, A. - Soldi, A., Le nuove riforme del processo civile (Padova, 2009), p. 222, ritengono essenziale l'infungibilità sia del facere che del non facere per l'applicazione della tutela in esame. 
di "ritardo nell'esecuzione del provvedimento", si attaglia perfettamente anche alle obbligazioni di "distruggere", la soluzione più ampia appare da prescegliersi.

In conclusione, la finalità perseguita dalla norma in esame, introducendo l'esecuzione indiretta, appare duplice: da un lato, si è mirato ad introdurre una nuova forma di tutela per situazioni che erano prive di salvaguardia coattiva; dall'altro, a rafforzare la tutela esecutiva già esistente.

\section{IL SISTEMA INTRODOTTO DALL'ARTICOLO 614 BIS INCONTRA DUE ORDINI DI LIMITI, UNO GENERALE ED UNO SPECIALE}

In primo luogo, esso non trova applicazione con riferimento alle obbligazione di fare fungibile: infatti, come già si è detto, solo gli obblighi di fare infungibile (oltre agli obblighi di non fare), sono considerati dalla norma.

L'esclusione in questione appare difficile da giustificare su un piano logico, considerato che la nuova disciplina si applica ad altre obbligazioni fungibili, quali sono quelle di non fare. In ogni caso essa è destinata ad incidere sensibilmente dal punto di vista operativo, giacché pone in primo piano la distinzione tra obbligazioni fungibili e infungibili, molto chiara in astratto, ma non altrettanto nelle applicazioni pratiche: basti pensare al contrasto di opinioni verificatosi in dottrina e giurisprudenza quanto all'esecuzione coattiva dell'obbligo di reintegra nel posto di lavoro, appunto derivante dai contrasti sul carattere fungibile o infungibile di tale obbligo $^{12}$.

Oltre a questa limitazione di carattere generale, ne sussiste un'altra che riguarda specifiche controversie: la disciplina posta dall'articolo 614 bis c.p.c. non si applica, per espressa previsione del primo comma, alle controversie di lavoro subordinato pubblico e privato e ai rapporti di collaborazione continuativa e coordinata di cui all'articolo 409 c.p.c.

Si tratta di un'esclusione che è stata prevalentemente criticata, quando non sospettata di contrasto con la Costituzione ${ }^{13}$. In realtà, la scelta

${ }^{12}$ Per una rassegna degli orientamenti giurisprudenziali in proposito, v. TATARELLI, Il licenziamento individuale e collettivo (2a ediz., Padova, 2000), pp. 38 ss. Sul problema in generale, v. BORGHESI, Rapporto di lavoro ed esecuzione in forma specifica, in Studi di Diritto processuale civile in onore di Giuseppe Tarzia (Milano, 2005), II, pp. 1117 ss.; Proto PISANI, Licenziamento e reintegra nel posto di lavoro, in Nuovi studi di diritto processuale del lavoro (Milano, 1992), pp. 116 ss.

${ }^{13} \mathrm{Cfr}$. Bove, Brevi riflessioni sui lavori in corso nel riaperto cantiere della giustizia civile, in www.judicium.it (con riferimento al d.d.l.); CARRATTA, A., in MANDrioli, C. - Carratta, A., Come cambia il processo civile (Torino, 2009), pp. 101 s.; Man- 
legislativa, se sembra poter trovare una giustificazione in una valutazione a priori del carattere personalissimo delle prestazioni che costituiscono, normalmente, l'oggetto di tali controversie, prestazioni da sottrarre ad ogni forma di coercizione, è probabilmente andata oltre l'obiettivo perseguito, escludendo l'applicabilità della nuova norma ad ogni tipo di obbligazione derivante dai rapporti in oggetto: sicché appare condivisibile - in ragione del canone interpretativo secondo il quale, fra più interpretazioni, va prescelta quella conforme alla Costituzione - la lettura restrittiva proposta da una parte della dottrina, secondo la quale la limitazione andrebbe circoscritta alle controversie aventi ad oggetto "prestazioni di lavoro subordinato pubblico o privato e prestazioni di collaborazione coordinata e continuativa di cui all'articolo 409"14.

In ogni caso, la suddetta esclusione fornisce una conferma e pone un dubbio.

Quanto al primo aspetto, essa conferma il fatto che le obbligazioni derivanti dal rapporto di lavoro sono da considerarsi infungibili. Diversamente, non sarebbero state menzionate, visto che la previsione dell'articolo 614 bis c.p.c. non si applica alle obbligazioni di fare fungibile; sicché si ha un'ulteriore dimostrazione del fatto che la reintegra nel posto di lavoro non è realizzabile coattivamente.

Il dubbio, invece, concerne la portata dell'esclusione in parola: se, cioè, essa valga, o meno, anche per i rapporti agrari, di cui all'articolo 409, n. 2, c.p.c. Se si guarda alla lettera della norma, la risposta dovrebbe essere negativa, ma se si guarda alla sua ratio, quella affermativa si impone, non essendoci differenze tra detta ipotesi e le altre menzionate dalla disposizione in esame.

\section{L'AZIONE DI CONDANNA, AVENTE AD OGGETTO UN "FACERE" INFUNGIBILE. CONDANNA DIRETTA AD INIBIRE}

I presupposti per ottenere la pronuncia della condanna al pagamento di una somma di denaro, in caso di inosservanza della decisione del giudice, sono rappresentati, in primo luogo, dal fatto che venga esperita un'azione di condanna, avente ad oggetto un facere infungibile o l'eliminazione di

DRIOLI, ult. cit., p. 117; PROTO PISANI, La riforma del processo civile: ancora una legge a costo zero (note a prima lettura), in Foro it., 5 (2009), p. 223; Soldi, cit. (n. 11), p. 225. Secondo G. F. RicCI, G. F., cit. (n. 7), p. 91, nota 6, "l'esclusione è ovviamente giustificata dalla presenza delle analoghe misure previste dall'articolo 18 , quarto comma, legge n. 300 del 1970". V. anche, in senso non critico, SASSANI, B. - TisCINI, R., Prime osservazioni sulla legge 18 giugno 2009, n. 69, in www.judicium.it.

\footnotetext{
${ }^{14}$ Carratta, cit. (n. 13), pp. $102 \mathrm{s.}$
} 
quanto realizzato in violazione di un'obbligazione di non fare; o, ancora, una condanna diretta ad inibire (ulteriori) comportamenti in violazione di un obbligo di astensione.

Nell'ambito dell'azione di condanna, la pronuncia prevista dall'articolo 614 bis c.p.c. avrà natura accessoria e non potrà essere pronunciata d'ufficio, ma dovrà essere sempre richiesta dalla parte. Tale istanza va formulata nell'atto introduttivo del giudizio, concretando una domanda vera e propria, ancorché accessoria. Essa potrà essere contenuta anche nella comparsa di risposta, qualora acceda ad una riconvenzionale proposta dal convenuto.

È da escludersi, invece, che la condanna accessoria possa essere richiesta laddove ci si limiti a promuovere un'azione di mero accertamento o si agisca per ottenere una pronuncia costitutiva, giacché l'articolo 614 bis c.p.c. áncora specificamente la pronuncia compulsiva accessoria all'esperimento di un'azione di condanna. Limitazione che ha una precisa logica, perché nel secondo caso il risultato si ottiene indipendentemente dalla collaborazione dell'obbligato, mentre nel primo è la stessa parte a circoscrivere la sua richiesta ad una pronuncia che individui i rispettivi obblighi, prescindendo totalmente dai profili e dalle conseguenze del loro inadempimento.

La pronuncia accessoria di condanna potrà essere contenuta in un "provvedimento" di condanna: non solo nella sentenza ${ }^{15}$, quindi, ma anche in altri atti ${ }^{16}$, come l'ordinanza di condanna che conclude il procedimento sommario ${ }^{17}$.

La condanna compulsiva accessoria, se si rientra nell'ambito di applicazione dell'articolo 614 bis c.p.c. e ricorrono i presupposti di cui si è detto, dovrà sempre essere pronunciata ${ }^{18}$, a meno che sia manifestamente iniqua.

${ }^{15}$ Che potrà essere anche di appello, laddove il giudice di primo grado abbia erroneamente negato la pronuncia compulsiva accessoria. Conf. Bove, cit. (n. 13), $\$$ 15; Mandrioli, cit. (n. 6), p. 116; Soldi, cit. (n. 11), p. 225.

${ }^{16}$ Conf. MANDrIOLI, ibid.

${ }^{17}$ Non potrà, invece, essere contenuta in titoli esecutivi che non siano di formazione giudiziale, qual è il verbale di conciliazione, anche in sede giudiziale. Ciò non significa, ovviamente, che le parti non possano, di loro volontà, in occasione della concliazione, introdurre una penale per il caso di mancata esecuzione spontanea delle obbligazioni, anche infungibili, tra esse convenute, ma solo che non vi potrà essere, al di fuori di quanto pattuito tra le parti, una sanzione per l'inadempimento degli obblighi di fare infungibile e di non fare, che sia imposta dal giudice in forza della previsione dell'articolo 614 bis c.p.c. (così, invece, parrebbe Bove, cit. (n. 13), \$ 15 . Sul punto v. anche SolDI, cit. (n.11), p. 227.

${ }^{18}$ Osserva Ricci, G. F., cit. (n. 7), p. 91, che "la disposizione in ogni caso non lascia alcun margine discrezionale al giudice di decidere se applicare o meno la sanzione, ma gli impone l'obbligo di farlo", salva la manifesta iniquità. 
Si tratta di una valutazione che comporta una rilevante discrezionalità: tuttavia, costituendo l'eccezione rispetto alla regola, il rigetto dovrà essere adeguatamente motivato, tenendo presente che l'iniquità deve essere a tutti immediatamente percepibile, dovendo essere "manifesta".

Non è possibile, evidentemente, individuare un catalogo di situazioni che rendono manifestamente iniqua la pronuncia compulsiva accessoria: riterrei che questa situazione ricorra quando, ad es., la condanna implichi, per essere adempiuta, il fatto del terzo. In questi casi, infatti, l'adempimento sfugge alla volontà dell'obbligato e l'applicazione di una condanna compulsiva per indurlo ad un adempimento che esula dalla sua diretta sfera di azione, appare ingiusta ${ }^{19}$.

Il potere del giudice è ampiamente discrezionale anche quanto alla determinazione della somma dovuta per ogni violazione o inosservanza. La norma si limita ad indicare alcuni parametri che dovranno essere apprezzati per tale valutazione, rappresentati dal valore controversia, dalla natura della prestazione e dal danno quantificato o prevedibile; ad essi si aggiunge "ogni altra circostanza utile" (articolo 614 bis, $2^{\circ}$ co., c.p.c.), il che vale ad attribuire al giudice il potere di tener conto di qualsiasi fatto che gli possa apparire rilevante. Anche qui, l'esigenza di un'adeguata motivazione è evidente, pur tenendo presente la funzione dissuasiva - chiaramente voluta dal legislatore - della norma ${ }^{20}$.

La condanna compulsiva accessoria potrà essere determinata in varia maniera. Potrà trattarsi di una somma da versarsi una tantum (sarà il caso normale per le violazioni future), ma anche - come fa pensare la locuzione "per ogni ritardo" - di versamenti periodici, in funzione del protrarsi dell'inadempimento. Più dubbio è che possa trattarsi di una somma che aumenta in ragione del protrarsi del ritardo, giacché la norma sembra riferirsi alla determinazione di un'unica somma.

Tale condanna costituisce titolo esecutivo. La norma fa riferimento, in proposito, solo alle "somme dovute per ogni violazione o inosservanza", omettendo di menzionare "il ritardo nell'esecuzione del provvedimento", ma tale ellittico riferimento non pare tale da privare di valenza esecutiva il provvedimento del giudice in quest'ultimo caso, giacché, diversamente, le obbligazioni di fare infungibile rimarrebbero prive di tutela esecutiva. Il che, oltre ad essere contrario alla volontà legislativa, quale emerge dalla

${ }^{19}$ Secondo Mandrioli, cit. (n. 6), p. 116, causa di esclusione sarà anche l'"obbiettiva impossibilità di adempiere anche per ragioni di solidarietà umana e sociale".

${ }^{20}$ Che la condanna in questione non abbia una natura risarcitoria, ma una funzione compulsiva, è da tutti ammesso: cfr. CARRATTA, cit. (n. 13), p. 103; Soldi, cit. (n. 11), p. 223. 
relazione $^{21}$, appare incompatibile con tutta la struttura della previsione legislativa, indubbiamente finalizzata a salvaguardare in pari misura le prestazioni di non fare e quelle di fare infungibile ${ }^{22}$.

Detta condanna è tendenzialmente condizionata: o alla mancata esecuzione spontanea dell'ordine del giudice oppure al fatto delle future violazioni o inosservanze dello stesso. Essa postula, nel caso si tratti di dare esecuzione a quanto disposto dal giudice, la fissazione, da parte di questi, del termine entro il quale l'adempimento spontaneo deve avvenire ${ }^{23}$.

Trattandosi di condanna condizionata, potranno sorgere delle contestazioni circa la tempestività o la regolarità dell'adempimento, come circa le future violazioni o inosservanze; contestazioni che andranno risolte tramite l'opposizione all'esecuzione eventualmente instaurata in forza della condanna condizionata ${ }^{24}$.

L'ultimo aspetto da esaminare, concerne l'individuazione del beneficiario della condanna compulsiva accessoria pronunciata dal giudice. Il quesito ha ragione di porsi perché l'esperienza comparatistica ci propone, in proposito, due diversi modelli: quello francese, giusta il quale beneficiario dell'astreinte è la parte a favore della quale doveva essere effettuata la prestazione ineseguita, e quello tedesco, in cui, invece, ne beneficia l'erario.

Il nostro sistema pare aver preferito la prima soluzione ${ }^{25}$. Al di là del fatto che in questo senso si esprime la relazione $e^{26}$, che sia la parte ad essere beneficiaria della condanna del giudice risulta dal fatto che la condanna in questione è condizionata dalla sua istanza: previsione che sarebbe del tutto ingiustificata se beneficiario ne fosse un terzo, quale l'erario, giacché in simile ipotesi il provvedimento in questione dovrebbe essere di espressione officiosa. Questo rilievo è, poi, confortato dall'essere il provvedimento del giudice titolo esecutivo, cioè un provvedimento che rileva in un quadro di rapporti tra parti contrapposte.

\footnotetext{
${ }^{21}$ Nella relazione al d.d.l. n. 1441, sub articolo 54, si accomunano, nella prospettiva della valenza esecutiva, gli obblighi di fare infungibile e di non fare.

${ }^{22}$ Conf., se non erro, Soldi, cit. (n. 11), p. 223.

${ }^{23}$ Contra RicCI, G. F., cit. (n. 7), p. 90, perché secondo l'A. la determinazione da parte del giudice "potrebbe prestarsi al più ampio arbitrio".

${ }^{24}$ Così anche la relazione al d.d.1. n. 1441, sub articolo 54. Analogamente Bove, cit. (n. 13), \$15; SoldI, cit. (n. 11), p. 224.

${ }^{25}$ Conf. Bove, cit. (n. 13), $\$ 15$; LuISO, Prime osservazioni sul disegno Mastella, in www.judicium.it, $\$ 20$; SASSANI - TISCINI, cit. (n. 13), $\$ 22$.

${ }^{26}$ V. relazione al d.d.l. n. 1441, sub articolo 54, la quale individua nel "creditore" il beneficiario della condanna del giudice.
} 


\section{OBBLIGAZIONI AVENTI AD OGGETTO UN OBBLIGO DI NON FARE}

Il quadro odierno della tutela per le obbligazioni aventi ad oggetto un obbligo di non fare, appare caratterizzato da una duplice prospettiva: da un lato quella di rafforzare la tutela esecutiva, laddove esistente; dall'altro, di prevenire eventuali inadempimenti futuri.

La prima prospettiva concerne, in sostanza, solo le obbligazioni di "distruggere" e mira ad ottenere l'adempimento spontaneo tramite una sanzione pecuniaria che si aggiunge alla tutela esecutiva rappresentata dalla possibilità di far eseguire forzosamente la condanna all'eliminazione di quanto non avrebbe dovuto essere fatto. Non si tratta di un'aggiunta inutile, quando si consideri che i costi per l'esecuzione forzata di un obbligo di non fare, talvolta molto rilevanti, fanno carico, almeno in via di anticipazione, alla parte che promuove l'esecuzione (articolo 614 c.p.c.); sicché ottenere l'esecuzione spontanea della sentenza del giudice non è privo né di utilità, né di rilevanza.

Le due forme di tutela -la condanna all'eliminazione di quanto non avrebbe dovuto essere fatto e al pagamento della somma di denaro per il ritardo nell'esecuzione spontanea del provvedimento- potranno essere richieste cumulativamente; se non c'è l'adempimento spontaneo, sussisterà il diritto del creditore sia ad avere il pagamento della somma (che, in certa misura, oltre ad avere una valenza sanzionataria per l'inosservanza dell'ordine del giudice, potrà anche valere a costituire i fondi necessari per l'esecuzione), sia a promuovere l'esecuzione coattiva della condanna alla distruzione di quanto non avrebbe dovuto essere fatto. Per contro, se vi è l'adempimento spontaneo, non ci sarà né il pagamento della somma prevista dal giudice, né, tanto meno, l'esecuzione in forma specifica.

Quanto alla seconda prospettiva perseguita dal legislatore, quella di prevenire eventuali inadempimenti futuri, stabilendo preventivamente una sanzione pecuniaria per ogni violazione o inosservanza successiva, essa generalizza, in sostanza, il meccanismo dell'inibitoria, già previsto per specifiche materie. In questo caso il giudice fisserà una somma da versarsi per ogni inadempimento futuro.

Non vedrei ostacolo alla possibilità di cumulo di tale condanna con quella di risarcimento del danno: considerata la funzione sanzionatoria della previsione normativa, che mira a far sì che il destinatario dell'ordine si astenga per il futuro da determinati comportamenti, sembra ammissibile la coesistenza con la tutela risarcitoria.

Infine, va sottolineato che nulla osta a che i due meccanismi di cui si è detto (cioè la condanna ad adempiere, con contestuale sanzione per il caso di mancato adempimento spontaneo, da un lato, e la condanna per 
futuri inadempimenti, dall'altro) possano essere utilizzati entrambi: il giudice potrà stabilire una sanzione per la mancata esecuzione spontanea del proprio ordine e un'eventuale ulteriore sanzione per violazioni od inosservanze successive.

\section{DifFicoltà AD AMMETTERE LA POSSIBILITÀ DI RICHIEDERE IN VIA}

\section{SUBORDINATA LE DUE FORME DI TUTELA}

Il quadro odierno della tutela per le obbligazioni aventi ad oggetto un facere infungibile vede aggiungersi alla possibilità di ottenere il risarcimento del danno per l'inadempimento, quella di richiedere la condanna dell'obbligato all'adempimento, condanna assistita (salvo che il giudice la ritenga manifestamente iniqua) da una statuizione accessoria di pagamento di una somma di denaro, in caso di mancata ottemperanza spontanea, al fine di creare una coercizione indiretta per ottenere l'adempimento.

Continua, quindi, a fare difetto la tutela esecutiva diretta, rispetto a questo tipo di obbligazioni: fermo che la sanzione per la loro violazione si realizza, in linea di principio, tramite il risarcimento del danno conseguente all'inadempimento, si aggiunge, per il creditore, un'ulteriore possibilità di tutela, rappresentata dalla condanna prevista dall'articolo in esame.

Il problema posto da tale innovazione è rappresentato dall'esigenza di coordinamento tra le varie forme di tutela. Se, infatti, si ammettesse la loro utilizzazione cumulativa, vi sarebbe il rischio di un arricchimento ingiustificato del creditore. Ciò si verificherebbe, infatti, nel momento in cui esso, dopo aver chiesto ed ottenuto il risarcimento del danno per l'inadempimento, ottenesse altresì l'adempimento spontaneo.

Se si vuol evitare questo risultato, bisogna immaginare che la condanna al risarcimento del danno ${ }^{27}$ sia alternativa rispetto alla condanna alla prestazione infungibile (e all'accessoria condanna compulsiva, per il caso di mancato adempimento), secondo la regola posta dall'articolo 1453 c.c. Accogliendo questa prospettiva, la richiesta di condanna ad un facere infungibile, con contestuale richiesta di condanna al pagamento di una somma di denaro per il caso di ritardo nell'adempimento, sarà possibile solo qualora non sia stata domandata la risoluzione del contratto con il relativo risarcimento del danno. Se l'esecuzione spontanea segue, nulla quaestio. Ove, al contrario, questa faccia difetto, la parte avrà diritto al pagamento della somma fissata dal giudice, per il caso di mancato

${ }^{27}$ Per l'inadempimento, rimanendo naturalmente sempre possibile, anche nel caso di richiesta di condanna alla prestazione infungibile, chiedere il risarcimento del danno conseguente al ritardo già verificatosi. 
adempimento, e successivamente potrà, perdurando questo, chiedere il risarcimento del danno.

Ovviamente, la richiesta di risoluzione del contratto (con correlativo risarcimento del danno), preclude quella di adempimento, secondo quanto previsto dall'articolo $1453,2^{\circ}$ comma, c.c.; mentre, secondo quanto sancito dalla suddetta disposizione, la richiesta di adempimento non preclude che in corso di giudizio venga richiesta la risoluzione e i danni conseguenti.

Non vedrei, infine, difficoltà ad ammettere la possibilità di richiedere in via subordinata le due forme di tutela (condanna all'adempimento, con condanna compulsiva accessoria e, in subordine, la risoluzione con condanna al risarcimento del danno $)^{28}$ : mi sembra, anzi, l'opportuna salvaguardia per il caso in cui il giudice non conceda la condanna compulsiva accessoria, ritenendola manifestamente iniqua.

È stato autorevolmente sostenuto ${ }^{29}$ che la norma in esame consentirebbe di richiedere, nel caso di contratto preliminare di vendita, in via cumulativa la pronuncia della sentenza che trasferisca la proprietà ex articolo 2932 c.c. e, al contempo, la condanna a dare esecuzione all'obbligo infungibile di concludere il contratto. Ciò permetterebbe di non dover attendere il passaggio in giudicato della sentenza costitutiva, giacché "si avrà un capo condannatorio, assistito da astreinte, che indurrà il convenuto alla stipula immediata (con atto notarile nel caso di beni immobili) del contratto definitivo" 30 .

La conclusione, peraltro, non convince.

Da un lato lascia perplessi che si possa richiedere una duplice statuizione, di condanna e costitutiva, per la medesima finalità, con riguardo alla stessa situazione giuridica ${ }^{31}$; dall'altro - e il rilievo mi sembra determinante - non sembra qualificabile come infungibile un'obbligazione suscettibile di esecuzione in forma specifica, in via sostitutiva. Infatti, qui non viene in gioco la categoria astratta della fungibilità-infungibilità, bensì la stessa va determinata in funzione della tutela che il legislatore ha apprestato alle singole obbligazioni. Non si può dimenticare, allora, che l'articolo 2932 c.c. è inserito nella sezione intitolata "dell'esecuzione forzata in forma specifica"; e la circostanza che la norma in questione non configuri un caso di

${ }^{28}$ La giurisprudenza ammette tale formulazione subordinata: cfr. Cass., 26 agosto 1986, n. 5235; cfr. anche Cass., 19 gennaio 2005, n. 1077; Cass., 4 dicembre 1999, n. 13563.

${ }^{29}$ Consolo, Una buona "novella" al c.p.c.: la riforma del 2009 (con i suoi articoli 360 bis e 614 bis) va ben al di là della sola dimensione processuale, in Corr. giur. (2009), p. 741.

${ }^{30}$ Ibid.

${ }^{31}$ In proposito v. anche supra, $\$ 4$. 
esecuzione forzata in senso proprio, non toglie che il contratto preliminare possa produrre i suoi effetti, se non è adempiuto spontaneamente, tramite la pronuncia del giudice della cognizione e, quindi, l'attività sostitutiva di questi.

Mi sembra, piuttosto, che si possa chiedere, in via gradata, la pronuncia della sentenza che tenga luogo del contratto non concluso, ex articolo 2932 c.c., e, in subordine, per il caso che non ricorrano i requisiti richiesti per tale pronuncia dal primo comma dell'anzidetta disposizione, la condanna all'adempimento, assistita dalla condanna compulsiva accessoria (o anche viceversa, a seconda delle peculiarità delle singole situazioni).

\section{CONCLUSIONI}

La statuizione compulsiva accessoria, se contenuta nella sentenza di condanna di primo grado, sarà suscettibile di sospensione da parte del giudice d'appello, ex articolo 283 c.p.c., al pari di ogni altra statuizione $e^{32}$; se poi, nonostante la disposta sospensione, sarà confermata, l'obbligo di pagamento della somma diverrà attuale dal momento della sentenza di appello ${ }^{33}$.

Qualora, invece, la sospensione non sia accordata, ma la statuizione prevista dall'articolo 614 bis c.p.c. venga riformata, non vedrei difficoltà, secondo i principi generali, ad ammettere la ripetibilità delle somme che siano state versate in ragione della pronuncia compulsiva accessoria. Non sembra convincente la tesi contraria, secondo la quale, attesa la peculiare natura "compulsoria", il soggetto condannato sarebbe tenuto al "pagamento della penalità di mora pure ove il suo appello venisse infine accolto e la sentenza di primo grado riformata" 34 . Nonostante il carattere sanzionatorio della statuizione in questione, non si può pretendere l'adempimento rispetto ad una condanna che risulti, poi, infondata. Manca correlativamente il titolo per trattenere quanto percepito in forza della decisione riformata.

L'ultimo aspetto di cui darsi carico concerne la possibilità di interporre appello qualora la parte abbia dato esecuzione all'obbligo di fare infungibile, per evitare l'applicazione della statuizione compulsiva accessoria. Ci si può domandare, infatti, se tale adempimento non comporti acquiescenza, precludendo, così, l'impugnazione.

Il dubbio non ha tanto ragion d'essere per le sentenze che condannino a distruggere un bene, che sono titoli esecutivi - rispetto alle quali, quindi,

\footnotetext{
${ }^{32}$ Come sarà possibile che tale condanna venga pronunciata per la prima volta dal giudice d'appello, qualora sia stata erroneamente negata in prime cure.

${ }^{33}$ Conf. Consolo, cit. (n. 29), p. 742.

${ }^{34}$ Ibíd.
} 
l'esecuzione spontanea pacificamente non comporta un atto incompatibile con la volontà di avvalesi delle impugnazioni - ma con riferimento alle sentenze di condanna all'adempimento di un obbligo di fare infungibile, che di tale caratteristica sono sfornite, non sussistendo la possibilità di loro esecuzione forzata e che sono assistite solo da uno strumento di coercizione indiretta.

Alla luce degli orientamenti più rigorosi, che tendono a privare di ogni rilevanza l'elemento volontaristico ${ }^{35}$, la risposta parrebbe dover essere affermativa circa l'esistenza, nella fattispecie, dell'acquiescenza. Tuttavia, la conclusione sembra scarsamente convincente. Non si può trascurare che l'articolo $329,1^{\circ}$ co., c.p.c. parla di "atti incompatibili con la volontà di avvalersi delle impugnazioni ammesse dalla legge", sicché i comportamenti della parte rilevano in quanto siano univocamente indicativi di una volontà spontanea di accettare la sentenza; volontà spontanea ${ }^{36}$ che non sembra configurabile nel comportamento di chi dia esecuzione ad una sentenza che lo condanni a tenere un comportamento infungibile, per evitare una sanzione che, diversamente, lo colpirebbe.

[Recibido el 31 de diciembre de 2009 y aprobado el 15 de marzo de 2010].

\section{BiBLIOGRAFÍA}

BorghesI, Rapporto di lavoro ed esecuzione in forma specifica, in Studi di Diritto processuale civile in onore di Giuseppe Tarzia (Milano, 2005), II.

Bove, Brevi riflessioni sui lavori in corso nel riaperto cantiere della giustizia civile, in www.judicium.it

Carratta, A., in Mandrioli, C. - Carratta, A., Come cambia il processo civile (Torino, 2009).

Chiarloni, Misure coercitive e tutela dei diritti (Milano, 1980).

CONSOlo, Una buona "novella" al c.p.c.: la riforma del 2009 (con i suoi articoli 360 bis e 614 bis) va ben al di là della sola dimensione processuale, in Corr. giur. (2009).

FERrara, L., L'esecuzione processuale indiretta (Napoli, 1915).

LuIso, Prime osservazioni sul disegno Mastella, in www.judicium.it.

Mandrioli, Corso di diritto processuale civile (7a ediz., Torino, 2009), VII.

MandRIOLI, Sulla correlazione necessaria tra condanna ed eseguibilità forzata, in Riv. Trim. Dir. Proc. Civ. (1976).

PICARDI, Codice di procedura civile (4a ediz., Milano, 2008), I.

${ }^{35}$ In proposito, per un quadro delle varie opinioni, cfr. PICARDI, Codice di procedura civile (4a ediz., Milano, 2008), I, pp. 1612 ss.

${ }^{36}$ Consolo, cit. (n. 29), p. 741, osserva che più che spontanea, la volontà può definirsi "spintanea". 
PROTO PISANI, La riforma del processo civile: ancora una legge a costo zero (note a prima lettura), in Foro it., 5 (2009).

Proto Pisani, Licenziamento e reintegra nel posto di lavoro, in Nuovi studi di diritto processuale del lavoro (Milano, 1992).

Proto Pisani, Per un nuovo codice di procedura civile, in Foro it., 5 (2009).

PunzI, Novità legislative e ulteriori proposte di riforma in materia di processo civile, in Riv. Trim. Dir. Proc. Civ. (2008).

RiccI, G. F., Ancora novità (non tutte importanti, non tutte pregevoli) sul processo civile, in Riv. Dir. Proc. (2008).

RicCI, G. F., La riforma del processo civile (Torino, 2009).

Sassani, B. - Tiscini, R., Prime osservazioni sulla legge 18 giugno 2009, n. 69, in www. judicium.it.

Silvestri, TARUfFo, Esecuzione forzata, III: Esecuzione forzata e misure coercitive, in Enc. giuridica, XIII, con Postilla di aggiornamento a tale voce (2006).

Soldi, A., in Bucci, A. - Soldi, A., Le nuove riforme del processo civile (Padova, 2009).

TARUffo, L'attuazione esecutiva dei diritti: profili comparatistici, in Riv. Trim. Dir. Proc. Civ. (1988).

TAtarelli, Il licenziamento individuale e collettivo (2a ediz., Padova, 2000).

Vullo, L'esecuzione indiretta tra Italia, Francia e Unione europea, in Riv. Dir. Proc. (2004). 